\title{
Comparison of Methotrexate with Tripterygium wilfordii Hook F in the Management of Rheumatoid Arthritis Jianguo Yuan', Haiyan $\mathrm{Ye}^{2}$, Shilin $\mathrm{Li}^{2}$ and Limin Chen ${ }^{2,3 *}$
}

${ }^{1}$ Affiliated Hospital of Chengdu University, Chengdu, Sichuan, China

${ }^{2}$ Institute of Blood Transfusion, Chinese Academy of Medical Sciences and Peking Union Medical College, Chengdu, Sichuan, China

${ }^{3}$ Toronto General Research Institute, University Health Network, University of Toronto, Toronto, Canada

\section{Editorial}

Rheumatoid arthritis (RA) is a serious chronic debilitating health problem, affecting approximately $1 \%$ population. The chronic autoimmune disease is characterized by persistent synovitis, systemic inflammation, and presence of autoantibody. Rheumatoid arthritis can lead to joint damage, disability, decreased quality of life and life expectancy $[1,2]$. In this editorial, we compared the two most commonly used drugs, Methotrexate and Tripterygium wilfordii Hook $\mathrm{F}$ for the treatment of RA, particularly focusing on the mechanism of action, safety and adverse effects.

\section{Introduction to Methotrexate and Tripterygium wilfordii Hook $\mathrm{F}$}

Antimetabolite methotrexate (MTX) was initially used as a chemotherapeutic agent [3]. However, as an inhibitor of folate metabolism, MTX has been commonly used for the treatment of RA for several decades [4]. Tripterygium wilfordii Hook F (TwHF) is a Chinese herb. The extracts from the root of TwHF have been widely used in traditional Chinese medicine for hundreds of years for the treatment of various autoimmune/inflammatory diseases, particularly for the management of RA [5]. Several disease-modifying antirheumatic drugs (DMARDs) have been used clinically, among which is MTX [1]. Previous studies demonstrated that extract of TwHF is also an alternative DMARD to treat patients with RA. Although the detailed etiology of RA remains unknown, DMARDs have been shown to reduce synovitis and systemic inflammation and to improve joint function [1].

\section{Mechanism of treating rheumatoid arthritis with Methotrexate or Tripterygium wilfordii Hook F}

Although MTX and TwHF have become two of the prescribed DMARDs for the treatment of RA for years, the mechanism of action is still incompletely understood. A number of studies have been initiated to define its underlying mechanisms of action. In the peripheral blood mononuclear cells (PBMCs) of RA patients, the folate level is significantly up-regulated under inflammatory conditions, and RA patients treated with MTX restores folate metabolism to normal levels [6]. Spurlock et al. demonstrate that, through a JNK-dependent pathway, MTX restores the function of proteins leading to cell cycle checkpoint deficiencies in $\mathrm{T}$ cells obtained from patients with RA [7]. And their findings also support a hypothesis whereby MTX activates distinct pathways in $\mathrm{T}$ cells and fibroblast-like synoviocytes (FLSs) to inhibit the activation of nuclear factor $k \mathrm{~B}$ (NF- $k \mathrm{~B}$ ), a critical activator of inflammatory processes [8]. MTX can not only induce the production of reactive oxygen species (ROS), but also increase JNK activity and sensitivity to apoptosis in activated $\mathrm{T}$ cells. Further studies indicated that the MTX-induced apoptosis is due to the fact that MTX inhibits the reduction of dihydrobiopterin to tetrahydrobiopterin. Patients with RA treated with low-dose MTX have been shown to have elevated levels of the JNK target gene, jun. This finding indicates that
MTX can activate this pathway in vivo, contributing to exert its antiinflammatory effect in RA [9].

TwHF exerts its function by affecting inflammatory mediators and immune system. Triptolide is one of the major components of the ethyl acetate (EA) extracts of TwHF. Nitric oxide (NO) has been regarded as an important inflammatory mediator. It has been shown that NO production and inducible NO synthase (iNOS) gene expression and transcription are inhibited by the EA extract of TwHF and Triptolide. Previous data suggest that the EA extract of TwHF could exert its role in RA by reducing iNOS activity to inhibit NO production [10]. Numerous evidence demonstrates that the chondrocyte itself plays significant role in cartilage destruction by production of matrix metalloproteinases (MMPs). In stimulated chondrocytes, both TwHF extract and triptolide potently blocked mRNA and protein expression of proinflammatory cytokine-induced MMP-3 and MMP-13 partly by impairing the activating protein- 1 (AP-1) and NF- $k \mathrm{~B}$ binding activities. Therefore, it is reasonable to postulate that MMPs may be the novel targets of antiarthritic actions of TwHF [11]. TwHF extract has been shown to be able to lower the IgM and IgM-rheumatoid factor in peripheral blood mononuclear cells separated from patients with RA [12]. TwHF extract itself also plays an important role in promoting apoptosis of $\mathrm{T}$ or $\mathrm{B}$ cells and in inhibiting their proliferation, collectively decreasing inflammation triggered by these cells [5]. Mechanisms of action of MTX or TwHF in treating RA are obviously very complicated. Although some evidence indicated that MTX or TwHF treatment may affect the function of immune cells and secretion of immune mediators/ cytokines, further studies are clearly needed to establish the detailed mechanisms of action of MTX and TwHF.

\section{Safety and adverse effects}

Despite its widespread use, MTX is associated with a number of serious potentially fatal side effects: bone marrow suppression, liver cirrhosis and pulmonary diseases. With long-term use, the risk of liver fibrosis is of particular concern [13,14]. A meta-analysis by Conway et al demonstrated a twofold increased risk of elevated transaminases of varying severity in patients treated with MTX, although long-term liver toxicity was unable to assess due to the short duration of the included clinical trials and the limitation of liver biopsies [14]. Data from

*Corresponding author: Limin Chen, Toronto General Research Institute University Health Network, University of Toronto, Ontario M5G 1L6, Toronto, Canada, E-mail: limin.chen@utoronto.ca

Received August 27, 2015; Accepted August 30, 2015; Published September 03, 2015

Citation: Yuan J, Ye H, Li S, Chen L (2015) Comparison of Methotrexate with Tripterygium wilfordii Hook $\mathrm{F}$ in the Management of Rheumatoid Arthritis. J Bioanal Biomed 7: e132. doi:10.4172/1948-593X.1000e132

Copyright: (c) 2015 Yuan J, et al. This is an open-access article distributed under the terms of the Creative Commons Attribution License, which permits unrestricted use, distribution, and reproduction in any medium, provided the original author and source are credited. 
Citation: Yuan J, Ye H, Li S, Chen L (2015) Comparison of Methotrexate with Tripterygium wilfordii Hook F in the Management of Rheumatoid Arthritis. J Bioanal Biomed 7: e132. doi:10.4172/1948-593X.1000e132

Laohapand et al. indicated that treatment of RA patients who were exposed to hepatitis B virus (HBV) with long-term MTX was safe and not associated with hepatitis B reactivation [4]. Current available data prove the safety of short-term MTX treatment in patients with RA [14].

A number of in vivo studies have suggested that TwHF extract has been identified as a DMARD to treatment RA with multiple functions, particularly in suppressing various immune and inflammatory responses. The safety of TwHF extracts and their effects on the treatment of RA are systematically assessed by meta-analysis, demonstrating that TwHF extracts have the similar efficacy to other DMARDs in the treatment of RA [15]. However, although TwHF have been beneficial for patients with RA, subsequent adverse effects of TwHF have been reported and should not been ignored. TwHF may cause the malfunction of the male and female reproductive system. It is reported that gastrointestinal tract disturbances, diarrhea, leukopenia, thrombocytopenia, rash, and skin pigmentation are associated with the treatment with TwHF [5].

\section{Conclusion}

In summary, both MTX and TwHF have been identified as DMARDs used to treat RA with anti-inflammatory and immunosuppressive effects. TwHF was mainly approved in China. Although TwHF has toxic potential, careful extraction procedures can limit the of adverse reactions [16] and most of the adverse reaction could be resolved by dose adjustment [5]. The anti-inflammatory and immunosuppressive effect of TwHF is comparable with MTX. Moreover, TwHF combination with MTX may be better than TwHF or MTX monotherapy [17]. Although much work is still needed to be done, MTX combinations with TwHF or other DMARDs are effective in the management of patients with RA.

\section{References}

1. Scott DL, Wolfe F, Huizinga TW (2010) Rheumatoid arthritis. Lancet 376: 1094 1108.

2. Bartok B, Firestein GS (2010) Fibroblast-like synoviocytes: Key effector cells in rheumatoid arthritis. Immunol Rev 233: 233-255.

3. Jolivet J, Cowan KH, Curt GA, Clendeninn NJ, Chabner BA (1983) The pharmacology and clinical use of methotrexate. N Engl J Med 309: 1094-1104.

4. Laohapand C, Arromdee E, Tanwandee T (2015) Long-term use of methotrexate does not result in hepatitis $B$ reactivation in rheumatologic patients. Hepatol Int 9: 202-208.
5. Bao J, Dai SM (2011) A Chinese herb Tripterygium wilfordii Hook F in the treatment of rheumatoid arthritis: Mechanism, efficacy, and safety. Rheumatol Int 31: 1123-1129.

6. Blits M, Jansen G, Assaraf YG, van de Wiel MA, Lems WF, et al. (2013) Methotrexate normalizes up-regulated folate pathway genes in rheumatoid arthritis. Arthritis Rheum 65: 2791-2802.

7. Spurlock CF 3rd, Tossberg JT, Fuchs HA, Olsen NJ, Aune TM (2012) Methotrexate increases expression of cell cycle checkpoint genes via JNK activation. Arthritis Rheum 64: 1780-1789.

8. Spurlock CF 3rd, Gass HM 4th, Bryant CJ, Wells BC, Olsen NJ, et al. (2015) Methotrexate-mediated inhibition of nuclear factor ${ }^{\circ} \mathrm{B}$ activation by distinct pathways in T cells and fibroblast-like synoviocytes. Rheumatology (Oxford) 54: 178-187.

9. Spurlock CF 3rd, Aune ZT, Tossberg JT, Collins PL, Aune JP, et al. (2011) Increased sensitivity to apoptosis induced by methotrexate is mediated by JNK. Arthritis Rheum 63: 2606-2616.

10. B Wang, L Ma, X Tao, PE Lipsky (2004) Triptolide, an Active Component of the Chinese Herbal Remedy Tripterygium wilfordii Hook F, Inhibits Production of Nitric Oxide by Decreasing Inducible Nitric Oxide Synthase Gene Transcription. Arthritis Rheum 50: 2995-2303

11. Sylvester J, Liacini A, Li WQ, Dehnade F, Zafarullah M (2001) Tripterygium wilfordii Hook F Extract Suppresses Pro-inflammatory Cytokine-Induced Expression of Matrix Metalloproteinase Genes in Articular Chondrocytes by Inhibiting Activating Protein-1 and Nuclear Factor-kB Activities. Mol Pharmacol 59: 1196-1205.

12. Tao XL (1989) Mechanism of treating rheumatoid arthritis with Tripterygium wilfordii Hook. Effects on secretion of total IgM and IgM-rheumatoid factor by peripheral blood mononuclear cells. Zhongguo Yi Xue Ke Xue Yuan Xue Bao 11: $36-40$.

13. Whiting-O'Keefe QE, Fye KH, Sack KD (1991) Methotrexate and histologic hepatic abnormalities: A meta-analysis. Am J Med 90: 711-716.

14. Conway R, Low C, Coughlan RJ, O'Donnell MJ, Carey JJ (2015) Risk of liver injury among methotrexate users: A meta-analysis of randomised controlled trials. Semin Arthritis Rheum.

15. Liu Y, Tu S, Gao W, Wang Y, Liu P, et al. (2013) Extracts of Tripterygium wilfordii Hook $F$ in the Treatment of Rheumatoid Arthritis: A Systemic Review and Meta-Analysis of Randomised Controlled Trials. Evid Based Complement Alternat Med 2013: 410793.

16. Tao X, Younger J, Fan FZ, Wang B, Lipsky PE (2002) Benefit of an extract of Tripterygium wilfordii Hook $F$ in patients with rheumatoid arthritis: A doubleblind, placebo-controlled study. Arthritis Rheum 46: 1735-1743.

17. Lv QW, Zhang W, Shi Q, Zheng WJ, Li X, et al. (2015) Comparison of Tripterygium wilfordii Hook $\mathrm{F}$ with methotrexate in the treatment of active rheumatoid arthritis (TRIFRA): A randomized, controlled clinical trial. Ann Rheum Dis 74: 1078-1086. 